\title{
MÉTODO DE MONTESSORI APLICADO À DEMÊNCIA - REVISÃO DA LITERATURA
}

\author{
Daniela Filipa Soares BRANDÃOa, José Ignacio MARTín ${ }^{b}$
}

\section{RESUMO}

O método de Montessori foi aplicado inicialmente às crianças, mas atualmente aplica-se a pessoas com demência. O objetivo deste trabalho é fazer uma revisão sistemática da investigação sobre a eficácia desse método, utilizando a Medical Literature Analysis and Retrieval System Online (Medline) com as palavras-chave demência e método de Montessori. Foram selecionados 10 estudos, onde se verificam melhorias significativas na participação e no envolvimento construtivo e diminuição dos afectos negativos e do envolvimento passivo. Não obstante, as revisões da literatura acerca desta intervenção não farmacológica em demência classificam esse método como fraco, em termos de eficácia. Essa aparente discrepância pode explicar-se porque o método de Montessori pode ter, de fato, uma influência pouco significativa em dimensões como a dos problemas comportamentais ou porque não existe investigação acerca desse método com elevados níveis de controle como são a presença de vários grupos de controle ou o duplo-cego.

Descritores: Revisão. Demência. Idoso. Terapêutica.

\section{RESUMEN}

El método Montessori se aplicó inicialmente a los niños, pero ahora se aplica a las personas con demencia. El propósito de este estudio es revisar de forma sistemática la investigación sobre la eficacia de este método, utilizando Medical Literature Analysis and Retrieval System Online (Medline) con las palabras clave demencia y método Montessori. Se seleccionaron 10 estudios, que tienen mejoras significativas en la participación y en el compromiso constructivo, y la reducción de efectos negativos y de la participación pasiva. Sin embargo, la revisión de la literatura sobre esta intervención no farmacológica en la demencia clasifica este método como débil en términos de eficacia. Esta aparente discrepancia puede explicarse porque el método Montessori puede tener una influencia poco significativa en los problemas de conducta, o porque no hay investigación sobre este método con altos niveles de control como son la presencia de varios grupos de control o el estudio doble ciego.

Descriptores: Revisión. Demencia. Anciano. Terapéutica

Título: Método Montessori aplicado a la demencia - revisión de la literatura.

\section{ABSTRACT}

The Montessori method was initially applied to children, but now it has also been applied to people with dementia. The purpose of this study is to systematically review the research on the effectiveness of this method, using Medical Literature Analysis and Retrieval System Online (Medline) with the keywords dementia and Montessori method. We selected 10 studies, in which there were significant improvements in participation and constructive engagement, and reduction of negative affects and passive engagement. Nevertheless, systematic reviews about this non-pharmacological intervention in dementia rate this method as weak in terms of effectiveness. This apparent discrepancy can be explained because the Montessori method may have, in fact, a small influence on dimensions such as behavioral problems, or because there is no research about this method with high levels of control, such as the presence of several control groups or a double-blind study.

Descriptors: Review. Dementia. Aged. Therapeutics

Title: Montessori Method applied to dementia - Literature review.

a Gerontóloga, Mestre em Gerontologia, ramo de Gestão de Equipamentos Sociais, bolseira de Investigação na Unidade de Investigação e Formaça sobre Adultos e Idosos (UnIFai), Porto, Portugal.

b Psicólogo, Doutor em Ciências Biomédicas, Professor auxiliar na Secção Autónoma das Ciências da Saúde da Universidade de Aveiro. Membro da Unidade de Investigação e Formação sobre Adultos e Idosos (UnIFai). Aveiro, Porto, Portugal 


\section{INTRODUÇÃO}

O método de Montessori foi criado por Maria Montessori (1870-1953) para ajudar crianças com dificuldades e considera a aprendizagem como um processo natural, que se desenvolve através da interacção com o ambiente ${ }^{(1-2)}$. As actividades Montessori envolvem a decomposição das tarefas, a provisão de materiais para manipular e o uso de deixas externas, adequando as tarefas às capacidades do indivíduo, diminuindo desse modo a probabilidade de erro e frustração ${ }^{(2)}$. Uma das capacidades mais requisitadas neste método é a memória processual ${ }^{(3)}$. Esta ainda se encontra relativamente preservada nos idosos com demência, comparativamente com outras capacidades cognitivas, o que defende a aplicação desta prática na população com Doença de Alzheimer ${ }^{(3)}$.

A operacionalização do método de Montessori engloba um conjunto de actividades que exploram cinco áreas distintas, sendo estas as actividades de vida diária, as experiências sensoriais, a matemática e raciocínio, a linguagem e as ciências ${ }^{(2,4)}$. As actividades baseadas neste método seguem ainda um conjunto de princípios de reabilitação que permitem que esta prática seja aplicada a pessoas com demência ${ }^{(5-6)}$. É importante utilizar materiais da vida real e esteticamente agradáveis, desenvolver actividades do simples para o complexo, progredir do concreto para o abstracto, permitir o progresso da aprendizagem e sequência (observação, reconhecimento, recordação/demonstração), utilizar o menos possível a vocalização quando são apresentadas as actividades e fazer pausas nas actividades e praticar uma tarefa de cada vez ${ }^{(2,6-7)}$. Atendendo a estas características, os investigadores têm aplicado com sucesso os princípios deste método para programar actividades estruturadas para indivíduos com demência ${ }^{(2)}$. A avaliação da sua eficácia em aspectos como a agitação e o envolvimento destes indivíduos com demência tem suscitado o interesse da comunidade científica ${ }^{(2,7)}$.

Esta revisão da literatura pretende analisar os principais estudos empíricos sobre o método de Montessori aplicado à demência. Apesar de a literatura baseada em provas indicar uma baixa evidência científica deste método, os estudos empíricos apresentam melhorias significativas no envolvimento e participação dos indivíduos ${ }^{(8)}$. Este estudo poderá dar algumas indicações para explicar esta discrepância, daí a sua importância.

\section{MÉTODOS}

A recolha de dados para esta revisão integrativa foi realizada através da base de dados Medical Literature Analysis and Retrieval System Online (Medline). Esta abrange um número elevado de artigos científicos, designadamente na área da saúde e, mais especificamente, da demência. A revisão integrativa constitui um método de pesquisa que pretende avaliar criticamente e sintetizar as evidências disponíveis sobre o tema em estudo ${ }^{(9)}$. A presente revisão visou o horizonte temporal desde 1997 até 2010 e decorreu entre Dezembro de 2010 e Janeiro de 2011 , tendo sido utilizadas as palavras-chave método de Montessori e demência (dementia). A pesquisa, utilizando as supracitadas palavras-chave demonstrou um total de 16 estudos. Como critério de inclusão considerou-se o facto de os artigos serem estudos empíricos originais e abrangerem como participantes pessoas idosas. Tendo em conta estes critérios, os estudos disponíveis sobre a temática do método de Montessori aplicado à demência reduziram-se para 10 estudos.

\section{RESULTADOS}

Para analisar os 10 estudos que satisfizeram os critérios de inclusão foi elaborado um quadro síntese que abrangia: identificação do estudo, tipo de técnica, amostra, instrumentos e principais resultados/conclusões (Tabela 1).

Os estudos analisados foram classificados consoante o tipo de técnica utilizado. É possível verificar que a metodologia adoptada para avaliar o impacto das actividades Montessori difere entre os vários estudos. Existiam estudos comparativos ${ }^{(10-14)}$, estudos com programas intrageracionais ${ }^{(15-16)}$ e estudos com programas intergeracionais ${ }^{(4-5)}$. Um dos estudos adoptou ainda uma metodologia diferenciada, com um programa de treino dirigido a ajudantes familiares, que pretendia formar as profissionais para realizarem actividades com os utentes ${ }^{(17)}$.

Os estudos comparativos representam metade dos estudos $(\mathrm{n}=5)$ e comparam os efeitos das actividades Montessori com as actividades regulares da instituição, que serviam de controlo.

Os programas intrageracionais $(\mathrm{n}=2)$ fundamentam-se na atribuição do papel de líder a idosos com demência inicial, que conduzem as actividades Montessoricom idosos numa situação de demência moderada. Rentabilizam as capacidades remanescentes 
Tabela 1 - Principais estudos sobre o método de Montessori aplicado à demência na base de dados Medline, Portugal, 1997-2010.

\begin{tabular}{|c|c|c|c|c|}
\hline & Camp et al (1997) & $\begin{array}{l}\text { Orsulic - Jeras et al } \\
\text { (2000a) }\end{array}$ & $\begin{array}{l}\text { Gorzelle } \text { et al } \\
\text { (2003) }\end{array}$ & Camp et al (2004) \\
\hline Tipo de técnica & $\begin{array}{l}\text { Programa } \\
\text { intergeracional: } \\
\text { Idosos com demên- } \\
\text { cia e crianças idade } \\
\text { pré-escolar }\end{array}$ & $\begin{array}{l}\text { Estudo } \\
\text { Comparativo: } \\
\text { Atividades Mon- } \\
\text { tessori vs ativida- } \\
\text { des regulares }\end{array}$ & $\begin{array}{l}\text { Programa de treino: } \\
\text { Ajudantes familia- } \\
\text { res de utentes com } \\
\text { demência }\end{array}$ & $\begin{array}{l}\text { Programa } \\
\text { intrageracional: } \\
\text { Idosos com demência } \\
\text { inicial líderes de ativi- } \\
\text { dades com idosos com } \\
\text { demência avançada. }\end{array}$ \\
\hline Amostra & $\begin{array}{l}12 \text { idosos com } \\
\text { demência } \\
\text { M Idades: } 88 \text { anos }\end{array}$ & $\begin{array}{l}16 \text { idosos demência } \\
\text { avançada } \\
\text { M idades: } 88 \text { anos }\end{array}$ & $\begin{array}{l}\text { Sem amostra iden- } \\
\text { tificada }\end{array}$ & $\begin{array}{l}\text { Líderes: } 4 \text { idosos de- } \\
\text { mência inicial } \\
\text { Idades: } 74-91 \text { anos } \\
\text { Jogadores: } 7 \text { idosos } \\
\text { demência avançada; } \\
\text { Idades: } 82-95 \text { anos }\end{array}$ \\
\hline Instrumentos & $\begin{array}{l}\text { (i) } \mathrm{MMSE}^{*} \\
(\mathrm{M}=18) \\
\text { (ii) } \mathrm{DAFS}^{+}\end{array}$ & $\begin{array}{l}\text { i) } \operatorname{MMSE}^{*}(\mathrm{M}=6,1) \\
\text { (ii) } \mathrm{MOSES}^{+} \\
\mathrm{M}=100 \\
\text { (iii) } \mathrm{CMAI}^{\S}: \mathrm{M}=24 \\
\text { (iv) } \mathrm{CSD}^{\|} \mathrm{M}=5,9 \\
\text { (v) } \mathrm{MMP} / \mathrm{MAS}^{\boldsymbol{q}^{*}} \\
\text { (vi) } \mathrm{ARS}^{* *}\end{array}$ & $\mathrm{MPES}^{+\dagger}$ & $\begin{array}{l}\text { Líderes: (i) MMSE* } \\
\text { (M=23) } \\
\text { Jogadores: } \\
\text { (ii) MMSE* }(\mathrm{M}=8,3) \\
\text { (iii) } \mathrm{MPES}^{+\dagger} \\
\text { (iv) } \mathrm{ARS}^{* *}\end{array}$ \\
\hline \multirow[t]{2}{*}{$\begin{array}{l}\text { Resultados } \\
\text { principais }\end{array}$} & $\begin{array}{l}\text { Lições bem-sucedi- } \\
\text { das: M= } 22.3 \\
\text { (Mínimo } 10-\text { Má- } \\
\text { ximo 38) }\end{array}$ & $\begin{array}{l}\text { M M P / M A S : } \\
\text { Maior envolvimen- } \\
\text { to construtivo; Me- } \\
\text { nor envolvimento } \\
\text { passivo. } \\
\text { ARS: Menor ní- } \\
\text { vel de ansiedade e } \\
\text { medo. }\end{array}$ & $\begin{array}{l}\text { MPES: Maior en- } \\
\text { volvimento cons- } \\
\text { trutivo; Menor } \\
\text { envolvimento pas- } \\
\text { sivo; Maiores níveis } \\
\text { de prazer. }\end{array}$ & $\begin{array}{l}\text { Assistência do staff } \\
\text { não foi requerida pelos } \\
\text { líderes. } \\
\text { MPES: Maior envol- } \\
\text { vimento construtivo; } \\
\text { Maiores níveis de pra- } \\
\text { zer. }\end{array}$ \\
\hline & $\begin{array}{l}\text { Skrajner et al } \\
(2007)\end{array}$ & $\begin{array}{l}\text { Lee, Camp \& } \\
\text { Malone, }(2007)\end{array}$ & Lin et al. (2009) & $\begin{array}{l}\text { Gitoux, Robichaud } \\
\text { \& Paradis (2010) }\end{array}$ \\
\hline Tipo de técnica & $\begin{array}{l}\text { Programa } \\
\text { intrageracional: } \\
\text { Idosos com demên- } \\
\text { cia moderada con- } \\
\text { duzem atividade } \\
\text { de leitura para ido- } \\
\text { sos com demência } \\
\text { avançada. }\end{array}$ & $\begin{array}{l}\text { Programa } \\
\text { intergeracional: } \\
\text { Idosos com demên- } \\
\text { cia e crianças idade } \\
\text { pré-escolar. }\end{array}$ & $\begin{array}{l}\text { Estudo } \\
\text { Comparativo: } \\
\text { Atividades Mon- } \\
\text { tessori vs variação } \\
\text { da acupunctura vs } \\
\text { atividades regulares }\end{array}$ & $\begin{array}{l}\text { Estudo Comparativo: } \\
\text { Atividades Montessori } \\
\text { vs condição de con- } \\
\text { trolo }\end{array}$ \\
\hline
\end{tabular}


Continução.

\begin{tabular}{|c|c|c|c|c|}
\hline Amostra & $\begin{array}{l}\text { Líderes: } 6 \text { idosos } \\
\text { demência moderada } \\
\text { Midades: } 84,8 \text { anos } \\
\text { Participantes: } 22 \\
\text { idosos demência } \\
\text { moderada/avançada } \\
\text { Idade } \geq 60 \text { anos }\end{array}$ & $\begin{array}{l}\text { 14 idosos com diag- } \\
\text { nóstico de demência } \\
\text { M idades: } 90,29 \\
\text { anos }\end{array}$ & $\begin{array}{l}133 \text { indivíduos } \\
\text { institucionalizados } \\
\text { com demência. M } \\
\text { idades: } 80,1 \text { anos } \\
\text { Sequências: } \\
1(\mathrm{n}=42) ; 2 \quad(\mathrm{n}= \\
39) ; 3(\mathrm{n}=52)\end{array}$ & $\begin{array}{l}14 \text { idosos com demên- } \\
\text { cia moderada a severa }\end{array}$ \\
\hline Instrumentos & $\begin{array}{l}\text { Líderes: (i) MMSE* } \\
\text { (M=17,5) } \\
\text { Participantes: } \\
\text { (i ) M M S E * } \\
\text { (M=15,73) } \\
\text { (ii) QAR Leader } \\
\text { Assessment Form+ }++ \\
\text { (iii) MPES }{ }^{+\dagger} \\
\text { (iv) Entrevista } \\
\text { estruturada }\end{array}$ & $\begin{array}{l}\text { (i) MMP/MAS } \\
\text { (ii) MRI-ES } \$\end{array}$ & $\begin{array}{l}\text { (i) CMAI } \\
\text { (ii) Ease-of- Care } \\
\text { (iii) AARS }\|।\|\end{array}$ & $\begin{array}{l}\text { (i) Observação directa } \\
\text { e indirecta } \\
\text { (ii) DMPT } \\
\text { (iii) CMAI } \\
\text { (iv) Escala de Obser- } \\
\text { vação }\end{array}$ \\
\hline
\end{tabular}

\begin{tabular}{|c|c|c|}
\hline $\begin{array}{l}\text { Resultados } \\
\text { principais }\end{array}$ & $\begin{array}{ll}\text { MPES: Maior en- } & \text { M M P / M A S : } \\
\text { volvimento cons- } & \text { Maior envolvimen- } \\
\text { trutivo e prazer; } & \text { to construtivo; me- } \\
\text { diminuição do não } & \text { nor envolvimento } \\
\text { envolvimento. } & \text { passivo. }\end{array}$ & $\begin{array}{ll}\text { CMAI: Diminuição } & \\
\text { de comportamen- } & \text { CMAI: menores sinais } \\
\text { tos agitados, agres- } & \text { de ansiedade, raiva e } \\
\text { sivos e fisicamente } & \text { medo. } \\
\text { não agressivos. } & \text { Maiores sinais de pra- } \\
\text { AARS: Mais afectos } & \text { zer e felicidade } \\
\text { positivos no grupo } & \\
\text { de intervenção. } & \end{array}$ \\
\hline & Lin et al. (2010) & van der Ploeg (2010) \\
\hline Tipo de técnica & $\begin{array}{l}\text { Estudo Comparativo: } \\
\text { Atividades Montessori vs condição } \\
\text { de controlo }\end{array}$ & $\begin{array}{l}\text { Estudo Comparativo: } \\
\text { Atividades Montessori vs intervenção } \\
\text { controlo }\end{array}$ \\
\hline Amostra & $\begin{array}{l}85 \text { idosos com demência } \\
\text { M idades: } 81,18 \text { anos }\end{array}$ & $\begin{array}{l}\text { Indivíduos com demência moderada } \\
\text { a severa }\end{array}$ \\
\hline Instrumentos & $\begin{array}{l}\text { (i) } \mathrm{MMSE}^{*} \\
\text { (ii) Índice de Barthel } \\
\text { (iii) EdFED*** } \\
\text { (iv) } \mathrm{MNA}^{+{ }^{+\dagger}}\end{array}$ & $\begin{array}{l}\text { (i) Escalas de Classificação } \\
\text { (ii) } \mathrm{CMAI}^{\S}\end{array}$ \\
\hline
\end{tabular}

\section{Resultados principais}

EdFED - menores níveis de dificuldade de alimentação; maior necessidade de assistência física e verbal nas refeições. (i) Actividades Montessori são eficazes no tratamento de comportamentos desafiantes em indivíduos com demência.

Fonte: Medline

*MMSE - Mini Mental State Examination

+DAFS - Direct Assesstment of Functional Status

${ }^{\ddagger}$ MOSES - Multidimensional Observational Scale for Elderly Subjects

¿MAI - Cohen-Mansfield Agitation Inventory

${ }^{\prime} \mathrm{CSD}$ - Cornell Scale for Depression

^MMP/MAS - Myers Menorah Park/ Montessori-Based Assessment

System

**ARS - Affect Rating Scale
${ }^{ \pm}$MPES - Menorah Park Engagement Scale

${ }^{+}$QAR - Question Asking Reading

$\$$ MRI-ES - Myers Research Institute Engagement Scale

III AARS - Aparent Affect Rating Scale

『DMPT - Dementia Mood-pictures test

${ }^{* * *}$ EdFED - Edinburgh Feeding Evaluation in Dementia

${ }^{+{ }^{+}+M N A}$ - Mini Nutricional Assessment 
dos indivíduos com demência inicial e permitem uma actividade com interesse para os indivíduos com demência severa.

Por fim, os programas intergeracionais $(n=2)$ treinam idosos com demência moderada para liderarem sessões de Montessori com crianças em idade pré-escolar. O objectivo é que idosos e crianças trabalhem juntos nas actividades, com assistência mínima do $\operatorname{staff}^{(5)}$.

As amostras consideradas nos estudos são usualmente pequenas, especialmente nos programas inter e intrageracionais, no entanto, as investigações mais recentes têm vindo a inverter essa tendência ${ }^{(12)}$. As amostras dos estudos considerados variam entre um mínimo de 11 indivíduos $^{(15)}$ e um máximo de 133 indivíduos ${ }^{(12)}$ que apresentam uma média de idades elevada ${ }^{(5,11)}$.

No que diz respeito aos critérios de selecção dos indivíduos, a maioria dos estudos considera o estado mental e funcional dos indivíduos ${ }^{(4-5,10-16)}$.

O método de Montessori é orientado para estádios mais avançados da demência ${ }^{(2,15,18)}$. Assim, os participantes apresentam normalmente um nível moderado ou severo de demência, com baixas pontuações na Mini Mental State Examination ${ }^{(2,5)}$.

A intensidade e duração das intervenções são díspares. Existem tanto intervenções mais prolongadas e menos intensas, que se operacionalizam num período compreendido entre dois a três meses, cerca de uma a duas vezes por semana ${ }^{(13)}$, como intervenções mais intensas, com frequência diária ${ }^{(10-11)}$. Relativamente à duração das sessões, estas variam entre um mínimo de quinze minutos ${ }^{(10)}$ e um máximo de quarenta e cinco minutos ${ }^{(11-12)}$.

Na maior parte dos estudos empíricos analisados, o objectivo principal era investigar os efeitos do método de Montessori em construtos como o nível de agitação, o afecto, a participação e o envolvimento do indivíduo ${ }^{(5,10-11 ; 13-15)}$. Num dos estudos analisados, avaliaram também o impacto das actividades Montessori na capacidade de alimentar-se em indivíduos com demência ${ }^{(12)}$.

O envolvimento ("engagement”) é um dos parâmetros de avaliação com maior preponderância nos estudos, constituindo uma dimensão fundamental na aplicação do método de Montessori, uma vez que este método não se fundamenta na concretização de actividades sem erro, mas sim na aquisição de interesse e empenho do indivíduo no desenvolvimento das actividades ${ }^{(19)}$. Para a avaliação deste parâmetro, os autores criaram escalas específicas
(Myers Research Institute Engagement Scale e Menorah Park Engagement Scale) que avaliam cinco tipos diferenciados de envolvimento (envolvimento construtivo, envolvimento passivo, envolvimento activo, auto-envolvimento e não envolvimento) $)^{(15)}$.

Usualmente é efectuada uma pré e pós avaliação do indivíduo, mas é pouco comum a existência de follow-up $p^{(11)}$. Normalmente o individuo é observado apenas durante a intervenção, no entanto, em alguns estudos, os comportamentos eram avaliados antes, durante e após a intervenção ${ }^{(5)}$. O acompanhamento do individuo durante outras actividades de vida diária é importante para perceber se as melhorias verificadas durante a intervenção se mantêm ${ }^{(2,5)}$.

Nos estudos comparativos, os principais resultados encontrados foram o aumento do envolvimento construtivo e menor envolvimento passivo, maiores níveis de prazer e participação e melhoria dos estados afectivos ${ }^{(5,10-11 ; 13-17)}$. Pode contribuir também para atenuar sinais de agitação e agressividade $^{(13-14)}$ e parece ser um método bem aceite pelos indivíduos com demência, o que pode justificar a diminuição dos afectos negativos ${ }^{(11)}$.

Nos programas intergeracionais, os indivíduos com demência conseguiram assumir o papel de orientadores/professores e apresentaram maiores níveis de envolvimento positivo ${ }^{(4-5)}$. Este tipo de programa permitia exercitar as capacidades remanescentes dos indivíduos com demência e o número de lições bem sucedidas aumentava ao longo da intervenção ${ }^{(4)}$.

Nos programas intrageracionais, os indivíduos com demência moderada demonstraram capacidade de liderança e orientação ${ }^{(2,15)}$. Conseguiram conduzir actividades para indivíduos com demência avançada e apresentaram maior envolvimento e satisfação com esse papel ${ }^{(15-16)}$. O que indica que os indivíduos com demência ligeira podem constituir um recurso adicional para a intervenção junto com indivíduos com demência mais avançada ${ }^{(2,19)}$. Porém, a atribuição do papel de líder ao idoso com demência deve respeitar a criação de materiais claros e fáceis de seguir, o controlo do ambiente, a simplificação dos procedimentos no decorrer da actividade e a concretização do treino num ambiente de vida real ${ }^{(16)}$.

\section{DISCUSSÃO DOS RESULTADOS}

A análise dos estudos incluídos nesta revisão demonstra algumas discrepâncias, mas também semelhanças entre eles. As principais semelhanças verificam-se ao nível dos critérios considerados 
para a seleção dos indivíduos, ao nível do tamanho das amostras (que são pequenas), nos indicadores escolhidos para avaliação do impacto (sintomas de agitação, afecto, envolvimento) e na opção metodológica de considerar dois momentos de avaliação e a existência de grupos de controlo.

Grande parte dos estudos ${ }^{(4-5 ; 10-16)}$ considera pertinente avaliar previamente a capacidade mental e/ou funcional dos indivíduos. Como critério de seleção consideravam o diagnóstico de demência, o grau de deterioração cognitiva e a inexistência de limitações funcionais graves. Esta prévia avaliação pretende identificar situações que impeçam a realização de determinadas atividades, nomeadamente pela existência de alterações sensoriais na visão e audição ${ }^{(2)}$. O reduzido tamanho das amostras consideradas pode estar associado ao carácter eminentemente individual desta intervenção, à dificuldade de operacionalizar grupos homogéneos de indivíduos com demência, assim como a dificuldade em integrar e estudar as atividades Montessori em instituições que têm outras atividades pré-definidas para indivíduos com demência.

Todos os estudos comparativos ${ }^{(10-14)}$ consideraram a existência de condição e/ou grupos de controlo e grupo experimental, que eram avaliados previamente e após a intervenção. Apenas um ${ }^{(10)}$ considerou a existência de um momento de avaliação de follow up (6 meses após o inicio da intervenção).

Os instrumentos de avaliação escolhidos para analisar o impacto das atividades nos constructos considerados, exigem normalmente a observação e/ ou o preenchimento pelo técnico. Esta metodologia pode ter associada uma maior subjetividade, sendo mais difícil assegurar a validade entre avaliadores. Contudo, uma vez que a intervenção ocorre junto de pessoas com demência, o autopreenchimento constituiria uma solução pouco viável fidedigna.

As principais discrepâncias entre os estudos analisados nesta revisão verificam-se ao nível da metodologia adotada, assim como na intensidade e duração das intervenções. A metodologia adotada para analisar os impactos das atividades Montessori difere nos estudos considerados. Existe uma prevalência de estudos comparativos, que comparam os impactos de atividades baseadas no método de Montessori com as atividades regulares da instituição onde é realizado o estudo.

Esta discrepância entre os vários estudos torna difícil avaliar a eficácia do método de Montessori. Não obstante, os estudos empíricos considerados nesta revisão parecem indicar que as actividades Montessori estão associadas a um aumento do envolvimento, participação e afectividade dos indivíduos, assim como a uma diminuição de sinais de agitação e agressividade. A aprendizagem sem erro e a natureza progressiva aumentam o sentido de controlo e permitem que o indivíduo ao atingir com sucesso determinado patamar, se sinta motivado a continuar. Desta forma, consegue-se justificar o maior envolvimento e humor positivo que é verificado durante as actividades Montessori. Durante a intervenção, é também potenciado o contacto com técnicos ou outros indivíduos, o que pode explicar o aumento da participação e interação do indivíduo. Este método pode ser motivador, uma vez que a aprendizagem ocorre por contacto com o ambiente e envolve a estimulação das dimensões funcional, cognitiva e relacional. Apresenta também a vantagem da fácil e rápida adequação da dificuldade das atividades às capacidades remanescentes dos indivíduos com demência e o facto de considerar um claro caracter terapêutico de estimulação cognitiva e de programação individualizada.

Contudo, é importante refletir com cautela sobre estes resultados. Enquanto os estudos empíricos demonstram benefícios do método de Montessori a vários níveis ${ }^{(10 ; 12 ; 15)}$, a literatura baseada em provas indica que a eficácia desta intervenção é reduzida ${ }^{(8)}$.

Esta disparidade pode estar relacionada com os constructos e medidas de resultados que são definidos para avaliar a sua eficácia ${ }^{(2)}$. Os estudos que revelam baixa evidência enfatizam os efeitos na depressão e agitação, que são pouco significativos. Os estudos que enfatizam o envolvimento, estados de afectividade, interacção social e competências cognitivas são os que revelam mais benefícios desta técnica. Os constructos avaliados de forma observacional (e.g. envolvimento, agitação) apresentam resultados mais representativos. Contudo, a avaliação observacional pode também ter associada uma maior subjetividade. Uma vez que as atividades Montessori têm uma componente eminentemente prática os primeiros impactos deste método sentem-se nestes constructos. Os impactos nos níveis de depressão possivelmente apenas serão encontrados considerando um momento de follow up após a intervenção. A uniformização dos constructos e dos instrumentos de avaliação a considerar na avaliação da eficácia deste método assume importância particular para uma análise global da eficácia deste método. 


\section{CONSIDERAÇÕES FINAIS}

Os estudos empíricos considerados nesta revisão reforçam que o método de Montessori constitui uma intervenção benéfica, nomeadamente na redução de sintomas comportamentais.

O número escasso de estudos empíricos sobre a eficácia do método de Montessori e a disparidade na metodologia adoptada tornam difícil averiguar a evidência científica deste método. Ainda assim, este método parece ser importante na intervenção junto de indivíduos com demência, podendo ser utilizado como complemento de outras intervenções não farmacológicas.

As pequenas amostras de alguns dos estudos, $\mathrm{e}$ o facto de na maioria deles a observação dos comportamentos ocorrer apenas durante a sessão Montessori e não também durante outras actividades diárias, constituem as principais limitações dos artigos considerados. Nesse sentido, novos estudos devem considerar amostras de maior dimensão e determinar qual a frequência e duração da intervenção necessária para assegurar resultados satisfatórios e qual a duração dos benéficos obtidos com a intervenção após o seu término. Para tal, é importante definir um protocolo de avaliação comum e posteriores estudos devem comparar a intervenção com método de Montessori com outras intervenções não farmacológicas, e estudar a possibilidade de utilizar as actividades Montessori como ferramenta de avaliação das capacidades remanescentes em indivíduos com demência.

\section{REFERÊNCIAS}

1 Gutek G. The Montessori method: the origins of an educational innovation: including an abridged and annotated edition of Maria Montessori's The Montessori method. Lanham: Rowman \& Littlefield; 2004.

2 Mahendra N, Kim E, Bayles K, Hopper T, Azuma T. Evidence-based practice recommendations for working with individuals with dementia: Montessori-based interventions. J. Med. Speech Lang. Pathol. 2006; 14(3):xiii-xxi.

3 Vance D, Johns RJ. Montessori improved cognitive domains in adults with Alzheimer Disease. Phys Occup Ther Geriatr. 2002;20(3/4):19-36.

4 Camp CJ, Judge KS, Bye CA, Fox KM, Bowden J, Bell M. et al.. An intergenerational program for persons with dementia using Montessori methods. Gerontologist. 1997;37(5):688-692.
5 Lee MM, Camp CJ, Malone ML. Effects of intergenerational Montessori-based activities programming on engagement of nursing home residents with dementia. Clin Interv Aging. 2007;2(3):477-483.

6 Bognar J. Montessori-Based Activities for Dementia: A Walk Down Memory Lane. Age in Action 2003; 18(4).

7 Camp CJ, Schneider N, Orsulic-Jeras S, Mattern J, McGowan A, Antenucci VM, et al.. Montessori-based activities for persons with dementia. Beachwood:Menorah Park Center for Senior Living; 2006. vol. 2.

8 Livingston G, Johnston K, Rosenheck A, Hsiao JA, Schneider LS, et al. Systematic review of psychological approaches to the management of neuropsychiatric symptoms of dementia. Am J Psychiatry. 2005;162(11):1996-2021.

9 Souza M, Silva M, Carvalho R. Revisão integrativa: o que é e como fazer?. Einstein. 2010; 8(1):102-106

10 Orsulic-Jeras S, Judge KS, Camp CJ. Montessori-based activities for long-term care residents with advanced dementia: effects on engagement and affect. Gerontologist. 2000;40(1):107-111.

11 Lin LC, Yang MH, Kao CC, Wu SC, Tang SH, Lin JG. Using acupressure and Montessori-based activities to decrease agitation for residents with dementia: a cross-over trial. J Am Geriatr Soc. 2009; 57(6):1022-9.

12 Lin LC, Huang Y, Su S, Watson R, Tsai B, Wu S. Using spaced retrieval and Montessori-based activities in improving eating ability for residents with dementia. Int J Geriatr Psy. 2010;25: 953- 959.

13 Ploeg ES, O'Connor DW. Evaluation of personalised, one-to-one interaction using Montessori-type activities as a treatment of challenging behaviours in people with dementia: the study protocol $\mathrm{f}$ a crossover trial. BMC Geriatr 2010;10:3.

14 Giroux D, Robichaud L, Paradis M. Using the Montessori approach for a clientele with cognitive impairments: a quasi-experimental study design. Int J Aging HuM Dev. 2010;71(1):23-41.

15 Camp CJ, Skrajner MJ. Resident-Assisted Montessori Programming (RAMP): training persons with dementia to serve as group activity leaders. Gerontologist. 2004;44(3):426-31.

16 Skrajner MJ, Camp CJ. Resident-Assisted Montessori Programming (RAMP): use of a small 
group reading activity run by persons with dementia in adult day health care and long-term care settings. Am J Alzheimers Dis Other Demen. 2007;22(1):27-36.

17 Gorzelle GJ, Kaiser K, Camp CJ. Montessori-based training makes a difference for home health workers and their clients. Caring 2003;22(1):40-2.
18 Vance DE, Porter RJ. Montessori methods yield cognitive gains in Alzheimer's day cares. Aging. 2000;24(3):1-22.

19 Camp C, Cohen-Mansfield J, Capezuti E. Use of nonpharmacologic interventions among nursing home residents with dementia. Mental health services in nursing homes 2002;53(11):1397-1401.

Endereço do autor / Dirección del autor / Author's address:

Daniela Brandão

Unidade de Investigação e Formação sobre Adultos

e Idosos

Instituto de Ciências Biomédicas Abel Salazar

Rua Jorge Viterbo Ferreira, 228

4050-313, Porto, Portugal

E-mail: daniela.brandao@unifai.eu 